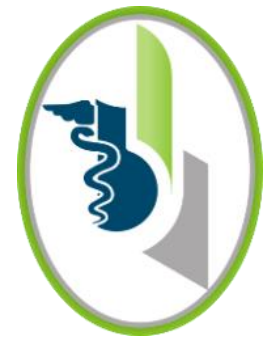

ACCESO ABIERTO

Para citaciones: Vargas, M., Cortés, E., Velázquez, J. (2020). Cambios moleculares en la remodelación cardiaca por síndrome metabólico. Revista Ciencias Biomédicas, 9(2), 131-146.

Recibido: 15 de mayo de 2020 Aprobado: 25 de junio de 2020

Autor de correspondencia: José Antonio Velázquez-Domínguez jauam14@yahoo.com.mx

Editor: Inés Benedetti. Universidad de Cartagena-Colombia.

Copyright: (C) 2020. Vargas, M., Cortés, E., Velázquez, J. Este es un artículo de acceso abierto, distribuido bajo los términos de la licencia https://creativecommons.org/licenses/by-nc-

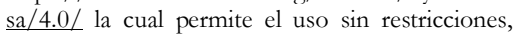
distribución y reproducción en cualquier medio, siempre y cuando el original, el autor y la fuente sean acreditados.

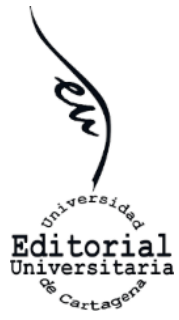

\section{Cambios moleculares en la remodelación cardiaca por síndrome metabólico}

\author{
Molecular changes in cardiac remodeling due to metabolic \\ syndrome.
}
Misael Vargas-López ${ }^{1}$ (D) Edgar Fernando Cortés-Martínez² iD , José Antonio Velázquez- Domínguez ${ }^{3}$ iD

${ }^{1}$ Escuela Nacional de Medicina y Homeopatía, Instituto Politécnico Nacional. CDMX, México

${ }^{2}$ Escuela Nacional de Medicina y Homeopatía, Instituto Politécnico Nacional. CDMX, México

${ }^{3}$ Posdoctorado, Centro de Investigación y Estudios Avanzados del IPN, Departamento de Infectómica y

Patogénesis Molecular, Escuela Nacional de Medicina y Homeopatía del IPN, CDMX, México.

\section{RESUMEN}

Introducción: el síndrome metabólico (SM) es un conjunto de anormalidades caracterizadas por el aumento de presión arterial, elevación de la glucemia en ayuno, dislipidemias, obesidad y/o diabetes, en donde se encuentran implicadas diversas moléculas que participan en la comunicación intracelular.

Objetivo: se describe la función y mecanismo de señalización intracelular que involucra a algunas de las moléculas que llevan a cabo procesos de remodelación cardiaca y son de especial interés como biomarcadores.

Métodos: se realizó una selección de literatura que nos describiera la participación de las moléculas en el proceso de remodelación cardiaca como resultado del SM.

Conclusiones: el SM y la falla cardiaca se encuentra íntimamente vinculados por la modulación de diversas vías de señalización intracelular que contribuyen a la remodelación cardiaca, generando cambios estructurales modificando la fisiopatología del cardiomiocito.

Palabras Clave: remodelación cardiaca; síndrome metabólico; obesidad; diabetes; dislipidemias; biología molecular.

\section{ABSTRACT}

Introduction: metabolic syndrome (MS) is a set of abnormalities characterized by increased blood pressure, elevated fasting blood glucose, dyslipidemia, obesity and I or diabetes, where various molecules that participate in intracellular communication are involved.

Objective: to describe the intracellular signaling function and mechanism that involves some of the molecules that carry out cardiac remodeling processes and are of special interest as biomarkers.

Methods: a selection of literature was carried out that described the participation of molecules in the process of cardiac remodeling as a result of the MS. 
Conclusions: MS and heart failure are closely linked by the modulation of various intracellular signaling pathways that contribute to cardiac remodeling, generating structural changes modifying the pathophysiology of the cardiomyocyte.

Keywords: cardiac remodeling; metabolic syndrome; obesity; diabetes; dyslipidemia; molecular biology.

\section{INTRODUCCIÓN}

Según la Organización Mundial de la Salud, el síndrome metabólico (SM) es un conjunto de anormalidades multisistémicas que se caracteriza por el aumento de la tensión arterial (HTA), diabetes mellitus tipo 2 (DM2), aumento del nivel sérico de triglicéridos, disminución del colesterol HDL, así como la presencia de obesidad abdominal.

En las últimas décadas el SM ha generado gran interés en el campo de la medicina al incrementarse exponencialmente las consecuencias que impactan negativamente en la calidad de vida de quienes lo padecen, dentro de las cuales tenemos afecciones cardíacas predominantes como el daño coronario, la hipertensión, los trastornos respiratorios del sueño y la insuficiencia cardíaca (IC) (1).

Durante la infancia y adolescencia, la obesidad representa un factor precursor muy importante que de no corregirse puede desencadenar complicaciones en la edad adulta y se relaciona con enfermedades cerebrovasculares, además de padecer remodelación cardiaca ( $\mathrm{RC}$ ), que se traducen en cambios en la estructura y la mecánica de las cámaras del corazón (2).

En el diabético se presentan afecciones producidas por la hiperglucemia e hiperlipidemia que causan estrés oxidativo cardíaco, disfunción endotelial, alteración del manejo del $\mathrm{Ca} 2+$ intracelular, así como disfunción mitocondrial (3); así pues, la coexistencia de estos trastornos en el paciente con SM eleva la mortalidad por afecciones cardiacas.

A nivel molecular, la RC se caracteriza por una respuesta inflamatoria responsable de incrementar los depósitos de matriz extracelular (MEC), y fibrosis; estos eventos podrían en última instancia promover disfunción diastólica y rigidez arterial como importantes alteraciones asociadas a la obesidad, y pueden facilitar el desarrollo de IC (4).

El propósito del presente manuscrito es revisar la participación de las diversas moléculas implicadas en la RC y que son de utilidad como factores pronósticos, dentro de las cuales la Galectina-3 (Gal3) y el factor de crecimiento transformante $\beta-1$ (TGF- $\beta 1$ ) desempeñan un papel importante para el depósito de MEC; la expresión de la proteína $G$ acoplada a receptor cinasa tipo 2 (GRK2) estimula la hipertrofia cardíaca al igual que p38 y ERK1/2, miembros de la familia de las proteínas cinasas activadas por mitógenos (MAPK). Moléculas antagonistas como la adiponectina que inhibe la RC y la leptina que la estimulan, también juegan un papel importante en la modificación de las estructuras cardíacas.

Existe además otro tipo de mecanismos que pueden resultar en disfunción del cardiomiocito, como la inhibición del peptidil-prolil isomerasa NIMA-1 (PIN1) que interfiere en la regulación celular normal, el factor nuclear-kappa $\beta(\mathrm{NF}-\kappa \beta)$ que contribuye en la protección contra la apoptosis de cardiomiocitos en hipoxia, o las Jun Cinasas (JNK) que participan en la proliferación celular y la modulación de la insulina.

\section{MÉTODOS}

Se realizó una selección y revisión de artículos recientemente publicados, entre enero de 2012 y mayo de 2020, que describieran el efecto de cada una de las moléculas participantes en el proceso de RC, considerando al SM como la antesala de este padecimiento. 


\section{RESULTADOS Y DISCUSIÓN}

\section{Participación de Galectina-3 y TGF- $\beta 1$}

Galectina-3 (Gal-3) es un miembro de la familia de las galectinas, un grupo de lectinas con la capacidad de unirse a $\beta$-galactósido (5). Gal-3 ha surgido como un mediador potencial del daño cardíaco debido a su capacidad para estimular la deposición de MEC y la amplificación de moléculas proinflamatorias (4).

Estudios clínicos han demostrado que los niveles de Gal-3 en sujetos con HA y DM2 fueron mayores que en el grupo control $(\mathrm{p}<0,05)$, esto posiblemente asociado a estrés cardiaco, niveles elevados de angiotensina II (Ang II) y sobrecarga de las cámaras cardiacas por presión elevada (6). Se sugiere que la secreción de Gal-3 está mediada por macrófagos, quienes representan un factor importante en la acumulación y activación de miofibroblastos, siendo estos últimos capaces de inducir fibrosis cardiaca. En conjunto, se puede concluir que la Gal- 3 juega un papel fisiopatológico importante en el desarrollo y progresión de la fibrogénesis y posterior falla cardiaca (FC) (Figura 1A) (5).

En los diabéticos la hiperglucemia y la hiperlipidemia crónica producen estrés oxidativo cardíaco, alteraciones metabólicas, remodelación de la MEC y fibrosis. Ocasionando manifestaciones denominadas miocardiopatía diabética (MCD) que implican anomalías estructurales y funcionales como hipertrofia ventricular izquierda, disfunción sistólica y diastólica, así como insuficiencia cardíaca y muerte (7).

Dado que la fibrosis juega un papel fundamental en MCD, TGF- $\beta$ es uno de los mediadores moleculares más estudiados (7); esta aumenta en respuesta a la lesión tisular en general y se encuentra involucrada en el proceso de reparación de tejidos y formación de cicatrices. Se sabe que los cardiomiocitos dañados liberan TGF- $\beta$ y Ang II en cantidades significativas en un evento postinfarto. La activación de TGF- $\beta$ en el corazón diabético también puede surgir como consecuencia de cambios en la expresión de algunos miRNA (microARN) así como de IncRNA (ARN largo no codificante) algunos estudios indican que estos modulan la vía de señalización de TGF- $\beta$ (7).

Además, se ha demostrado que tras agregar TGF- $\beta$ a cultivos de fibroblastos in vitro, se induce la expresión de genes relacionados con MEC y, por lo tanto, aumenta su deposición (Figura 1B.) y la supresión concomitante de la metaloproteinasa de matriz. (8) Los miofibroblastos (MF) son los principales mediadores de la producción de MEC. (9). En un estudio realizado en 72 pacientes con DM2 y nefropatía diabética se encontraron altos niveles de TGF- $\beta 1$ en orina y suero, lo que sugiere también una utilidad diagnóstica y pronóstica de la MCD (10).

\section{Papel de la peptidil-prolil isomerasa (PIN)}

Por su parte, se infiere que enzimas de la familia de las peptidil-prolil isomerasa (PIN) tienen un papel importante en la fibrosis cardiaca pues la inhibición de PIN1 disminuyó la inflamación de las vías respiratorias y el depósito de colágeno pulmonar en el asma, así como la fibrosis hepática inducida por dimetilnitrosamina (subproducto de procesos industriales y presente en niveles bajos en alimentos, ahumados o curados; probable carcinógeno en humanos) (9). Así mismo, algunos estudios han demostrado que PIN1 se encuentra altamente regulada en cardiomiocitos de ratones diabéticos, tras presentarse hipertrofia cardíaca inducida por sobrecarga de presión (9).

PIN1 está posiblemente ligada a la modulación de la vía TGF- $\beta$ /Smads pues el agotamiento de PIN1 regula negativamente los niveles de la proteína Smad $2 / 3$ y la señalización de TGF- $\beta$ (11), por lo que PIN1 resulta un blanco farmacológico atractivo en el que ya se han utilizado inhibidores específicos como la Junglona (compuesto orgánico extraído de la corteza del nogal negro, Juglans nigra) demostrando un efecto terapéutico positivo en la disfunción cardíaca y la fibrosis en ratones con MCD (9). 


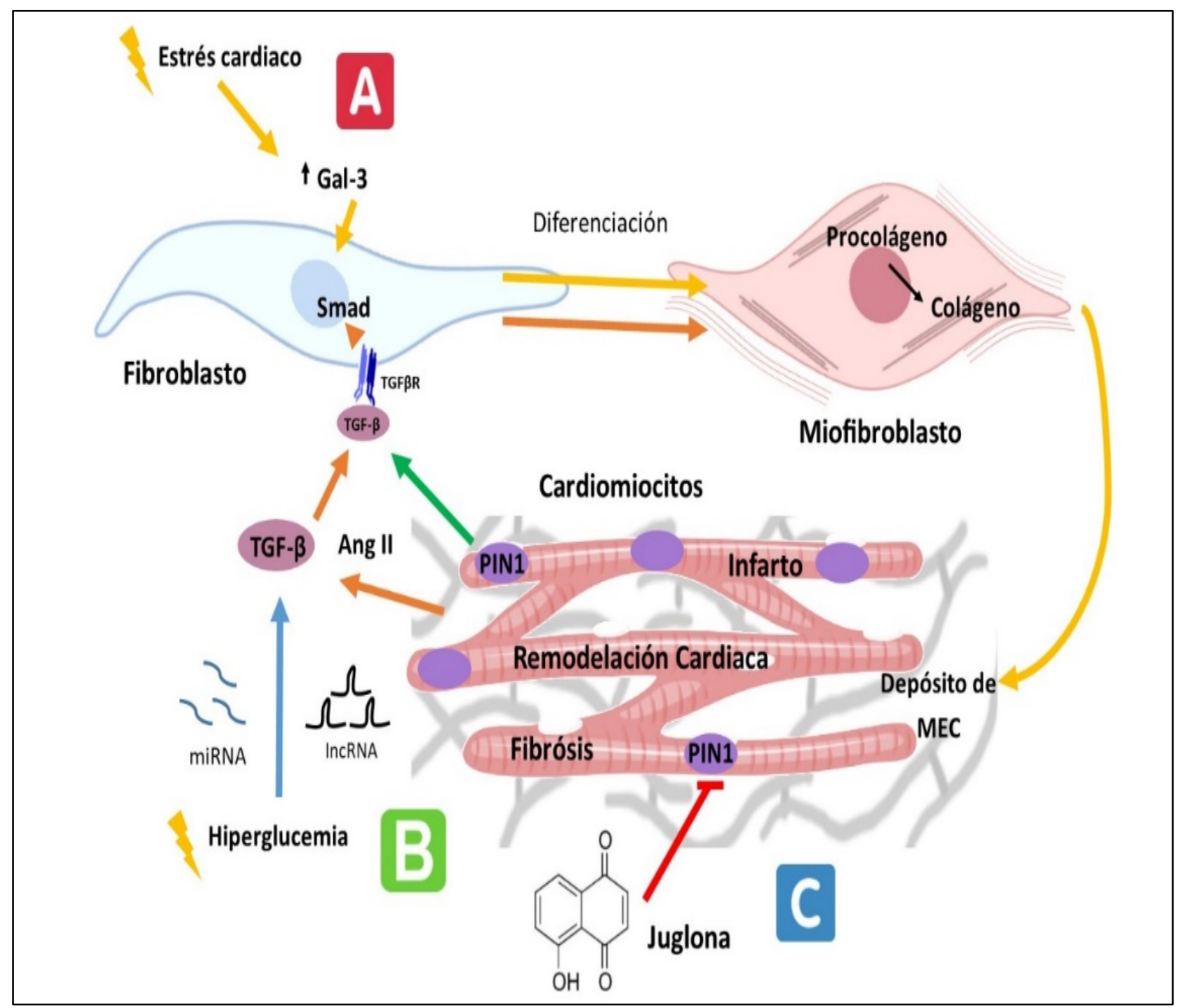

Figura 1. Participación de Gal-3, TGF- $\beta$ y PIN1 en la remodelación cardiaca. A. Gal-3 difunde la membrana plasmática interactuando con proteínas de la matriz extracelular (MEC), la progresión de esta molécula en los fibroblastos produce su diferenciación en miofibroblastos lo que induce fibrosis cardiaca (flecha amarilla); B. TGF- $\beta$ se expresa en respuesta a la lesión miocárdica (flecha naranja) y como consecuencia de la hiperglucemia a través de la regulación de miRNA y IncRNA (flecha azul), se acopla a su receptor TGF- $\beta$ R2 y fosforila al receptor de tipo 1, que se denomina ALK-4 (cinasa similar a activina). Esta cinasa fosforila y activa Smad2 o Smad3, que forma un heterodímero con Smad4, y se internaliza al núcleo regulando la expresión génica lo que provoca la diferenciación de fibroblastos a miofibroblastos y la consecuente producción de MEC; C. PIN1 (enzima nuclear) probablemente ligada a la regulación de la vía TGF- $\beta /$ Smad (flecha verde); se ha demostrado un efecto terapéutico positivo de la fibrosis cardiaca tras la inhibición de PIN1 con Juglona (flecha roja).

\section{Las MAPK en la hipertrofia cardiaca}

Las vías de señalización intracelular de la proteína cinasa activada por mitógenos (MAPK) son reguladores centrales de múltiples aspectos de la función celular en todos los tejidos, incluido el corazón (12). Existen cuatro subfamilias clásicas de
MAPK en humanos: ERK 1/2 (MAPK 3/1), p38 (MAPKs 11-14), JNK (MAPKs 8-10) y ERK5 (big MAPK 1 o MAPK 7) (3).

Las MAPK humanas responden a una variedad de estímulos diferentes, por ejemplo, ERK 1/2 y ERK5 
pueden ser desencadenadas por varios agonistas y citocinas. También se sabe que ERK $1 / 2$ se activa mediante factores de crecimiento; por otro lado, JNK y $\mathrm{p} 38$ pueden ser iniciados por diferentes factores de crecimiento y receptores acoplados a proteína $G$ (GPCR) (3).

Bajo estos estímulos de estrés se inician las cascadas de fosforilación conservadas en serie de tres niveles que incluyen una MAPK cinasa cinasa (MKKK), una MAPK cinasa (MKK) y una MAPK terminal.(13) Estudios de sobreexpresión conducen a activación simultánea de ERK, JNK y p38 MAPK en el corazón después de una sobrecarga de presión que contribuye al desarrollo de hipertrofia cardíaca patológica. En este modelo, la activación de ERK promueve el crecimiento de cardiomiocitos, JNK conduce a la formación reducida de uniones gap, p38 MAPK promueve la fibrosis cardíaca y la activación de las tres vías promueven una distensibilidad diastólica reducida (Figura 2). La sobrecarga de presión también conduce a la activación de la vía de la calcineurina A-NFAT en el corazón, que claramente desempeña un papel importante en la promoción del crecimiento de cardiomiocitos (13).

Particularmente p38 MAPK, desempeña un papel importante en la regulación de la $\mathrm{RC}$, entre las isoformas de p38 MAPK que se encuentran en el corazón sano, p38 $\alpha$ es la forma principal; en el que p38 $\beta$ muestra una expresión baja, y tanto p38 $\gamma$ como p38 son componentes menores. Existe evidencia de que p38 MAPK particularmente p38 $\alpha$ MAPK se activa durante la inflamación y el estrés oxidativo, la apoptosis, la hipertrofia y las anomalías del metabolismo energético (14).

Al igual que TGF- $\beta$, los estados hiperglucémicos pueden regular al alta o a la baja a distintos microARN que están implicados en la activación de p38 MAPK y la subsecuente aparición de cardiopatías, por ejemplo, la regulación alta de miR143 está asociada con la aparición de MCD; por el contrario, miR-24 tiene actividad cardioprotectora y su regulación a la baja implica apoptosis de cardiomiocitos en infarto y diabetes (14), (Figura 2A).
Por último, p38 a es importante para la proliferación, migración y diferenciación de fibroblastos cardíacos a miofibroblastos, se ha propuesto un papel para la inactivación de la fosfatasa DUSP1 / MKP1 (y por tanto la activación mejorada de p38 y ERK) como un mecanismo por el cual IL-17A estimula la proliferación $\mathrm{y}$ diferenciación de fibroblastos cardíacos mismos que son capaces de producir colágeno que se deposita en la MEC (12).

Las enfermedades cardiometabólicas (ECM) se caracterizan por un estado inflamatorio elevado, que promueven la enfermedad y comorbilidades. Las cinasas c-Jun N-terminal (JNK) se han descrito por su papel en la señalización de citocinas. Los datos han demostrado que las citocinas pueden activar la vía de señalización de JNK, pero también que la vía de JNK es responsable de la producción de citocinas. (15) (Figura 2C).

Cuando la homeostasis se desregula, tal como sucede en el SM, la activación de JNK es prolongada $\mathrm{y}$ deriva en inflamación crónica $\mathrm{y}$ apoptosis aberrante.

La primera evidencia del vínculo entre JNK y las ECM se demostró por la infiltración dependiente de JNK de células inmunes (macrófagos, células T, etc.), en el tejido adiposo y el hígado. Estos datos demostraron que los macrófagos son responsables de producir citocinas proinflamatorias como $\mathrm{TNF} \alpha \mathrm{e}$ IL6, lo que en última instancia da como resultado una alteración de la señalización de la insulina, resistencia a la insulina $y$ enfermedades cardiovasculares como arterioesclerosis, aneurisma aórtico abdominal, infarto agudo de miocardio e insuficiencia arterial periférica. En estas condiciones, JNK promovió la producción de citocinas a través de la transcripción génica impulsada por AP1 lo que desencadenó una inflamación crónica. Además, las quinasas JNK en conjunto con MLK y TAK1 promueven la producción de citocinas y la inflamación (15).

Esto podría resultar especialmente grave en sujetos obesos puesto que algunos estudios han demostrado que las JNK se activan en varios sitios 
metabólicamente importantes, por ejemplo, la activación de JNK en biopsias de músculo esquelético obtenidas de individuos obesos resistentes a la insulina fue mayor en comparación con los controles delgados. Además, biopsias de tejido adiposo subcutáneo han mostrado niveles aumentados de la forma activa fosforilada de JNK en individuos obesos en comparación con controles delgados (16).

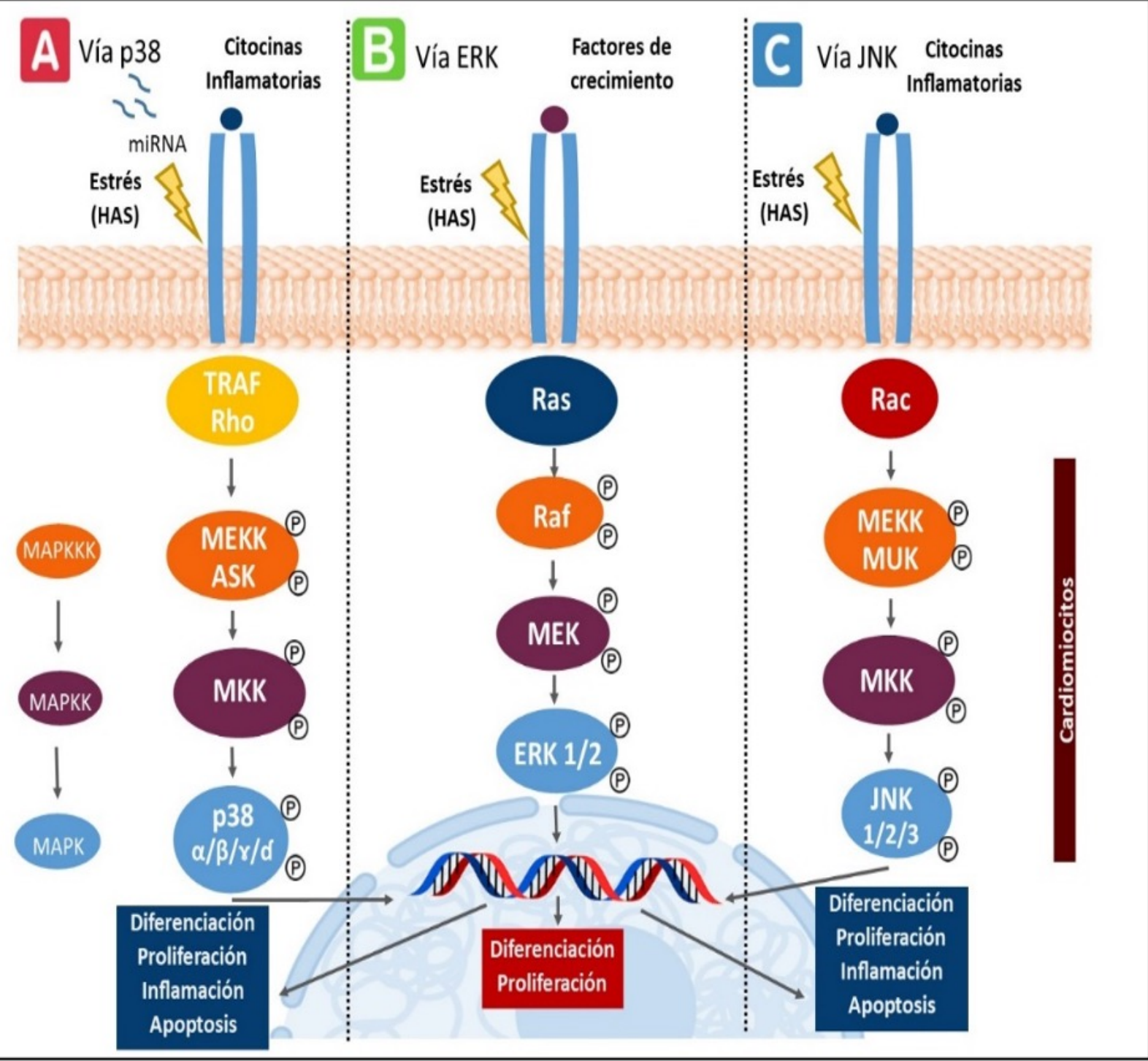

Figura 2. Modelo de activación simultanea de las MAPK y su repercusión en la IC. Las cascadas MAPK se organizan en tres niveles con la finalidad de promover la amplificación y fidelidad de la señal, modulando la respuesta hipertrófica del corazón a la sobrecarga de presión. A. La activación de la cascada MKKK-MKK3 / 6-p38, B. La activación de la cascada Raf-MKK-ERK1/2 participa en el crecimiento de cardiomiocitos individuales, y en el mecanismo de supervivencia después de una sobrecarga de presión, mientras que la activación de p38 $\alpha$ MAPK induce la disfunción contráctil de los cardiomiocitos. C. MKKK-MKK4/7- JNK antagoniza el crecimiento de cardiomiocitos individuales. La activación de p38 $\alpha$ MAPK y JNK1 / 2 promueve el remodelado cardíaco patológico como la apoptosis de cardiomiocitos en la zona del borde del infarto, expansión del infarto y fibrosis en el sitio del infarto y en el miocardio no afectado. 


\section{La GRK2 en la falla cardiaca}

Entre las múltiples vías anómalas en la IC, la señalización de proteína $\mathrm{G}$ acoplada a receptor (GPCR), se encuentra también desregulada. Un mecanismo crucial que encabeza la señalización de GPCR es la fosforilación de receptores unidos a ligandos a través de cinasas de GPCR (GRK). Los GRK inician un proceso, denominado "desensibilización del receptor", mediante la fosforilación de GPCR que conduce al reclutamiento de $\beta$-arrestina y a la internalización del receptor y regulación a la baja. En corazones humanos con IC, la expresión y actividad de GRK están elevadas. La GRK2 es la más abundante en el corazón, está regulada al alta, lo que lleva a aumento de la fosforilación, desensibilización y regulación a la baja de los GPCR clave, incluidos los receptores adrenérgicos $\beta$ ( $\beta \mathrm{AR}$ ) (Figura 3), (17).

La regulación al alza de GRK2 ocurre inicialmente después de una lesión cardíaca y es necesario para inhibir la sobreactivación de $\beta A R$ que se producen como resultado del incremento de catecolaminas en el corazón deteriorado por la activación del sistema nervioso simpático. Este es el comienzo del deterioro de la señalización adrenérgica en el que el exceso de norepinefrina y epinefrina se producen para compensar la disminución de la señalización de $\beta A R$ que finalmente mantiene la contractilidad disminuida (18).

\section{Adipocinas y cardiopatía}

La obesidad se caracteriza por el aumento depósito de tejido adiposo (TA) intraabdominal e intratorácico. En la actualidad el TA se considera un "órgano endocrino" con una intensa actividad metabólica. Las moléculas secretadas por el TA se denominan adipocinas y participan, no solo en el metabolismo energético, sino también en una amplia gama procesos fisiopatológicos (19), modulando la función del corazón y la vasculatura (Figura 4).

La mayoría de las adipocinas liberadas a partir del tejido adiposo, como el factor de necrosis tumoral $\alpha$ $(\mathrm{TNF} \alpha)$, la leptina, el inhibidor del activador del plasminógeno 1, la proteína fijadora de ácidos grasos de adipocitos, la lipocalina-2, la proteína quimiotáctica de los monocitos 1 y la resistina, ejercen efectos deletéreos sobre el sistema cardiovascular en particular el endotelio y el musculo liso vascular, así como en los cardiomiocitos. En la obesidad, la expansión del tejido adiposo conduce a la sobreproducción de estas adipocinas proinflamatorias, contribuyendo así a la patogénesis de las enfermedades cardiovasculares (ECV). Por otro lado, la adiponectina, una de las principales adipocinas secretadas por adipocitos con actividades antiinflamatorias y sensibilizantes a la insulina, está regulada negativamente en la obesidad lo que implica riesgo pues se ha estudiado ampliamente su papel en la protección cardiovascular (20).

La adiponectina regula la lesión cardiaca mediante la modulación de las reacciones antiinflamatorias e inhibiendo la RC.(21) Sus efectos están mediados por su potenciación de la acción de la insulina, el aumento de la producción de óxido nítrico, la estimulación de la síntesis de prostaglandinas por la inducción de ciclooxigenasa-2 (COX2) y la activación de la adenosina monofosfato, que también activa la proteína cinasa e inhibe la hipertrofia estimulada por los receptores $\alpha$ - adrenérgicos de miocitos cardiacos (21).

Por su parte la leptina estimula la hipertrofia cardíaca directamente a través de mecanismos de señalización celular e indirectamente a través de sus efectos sobre la hipertensión y el sistema nervioso simpático (SNS). Las acciones generales de la leptina incluyen la estimulación de la reacción inflamatoria, el estrés oxidativo, la aterogénesis y la trombosis, lo que promueve la disfunción endotelial, la rigidez arterial, el desarrollo y la vulnerabilidad de las placas ateroscleróticas,(22) ejerciendo un efecto inotrópico negativo sobre los cardiomiocitos a través de la producción endógena de óxido nítrico (21).

Otras adipocinas como la resistina y visfatina podrían estar involucradas en la patogenia de ECV además de la resistencia a la insulina, DM2 y enfermedad renal crónica.(22) El papel de la resistina en patologías cardiacas y la lesión de isquemia-reperfusión aún no se ha definido, aunque 
se ha relacionado con mecanismos de dislipidemia e inhibición de la transportación vesicular de glucosa (21).

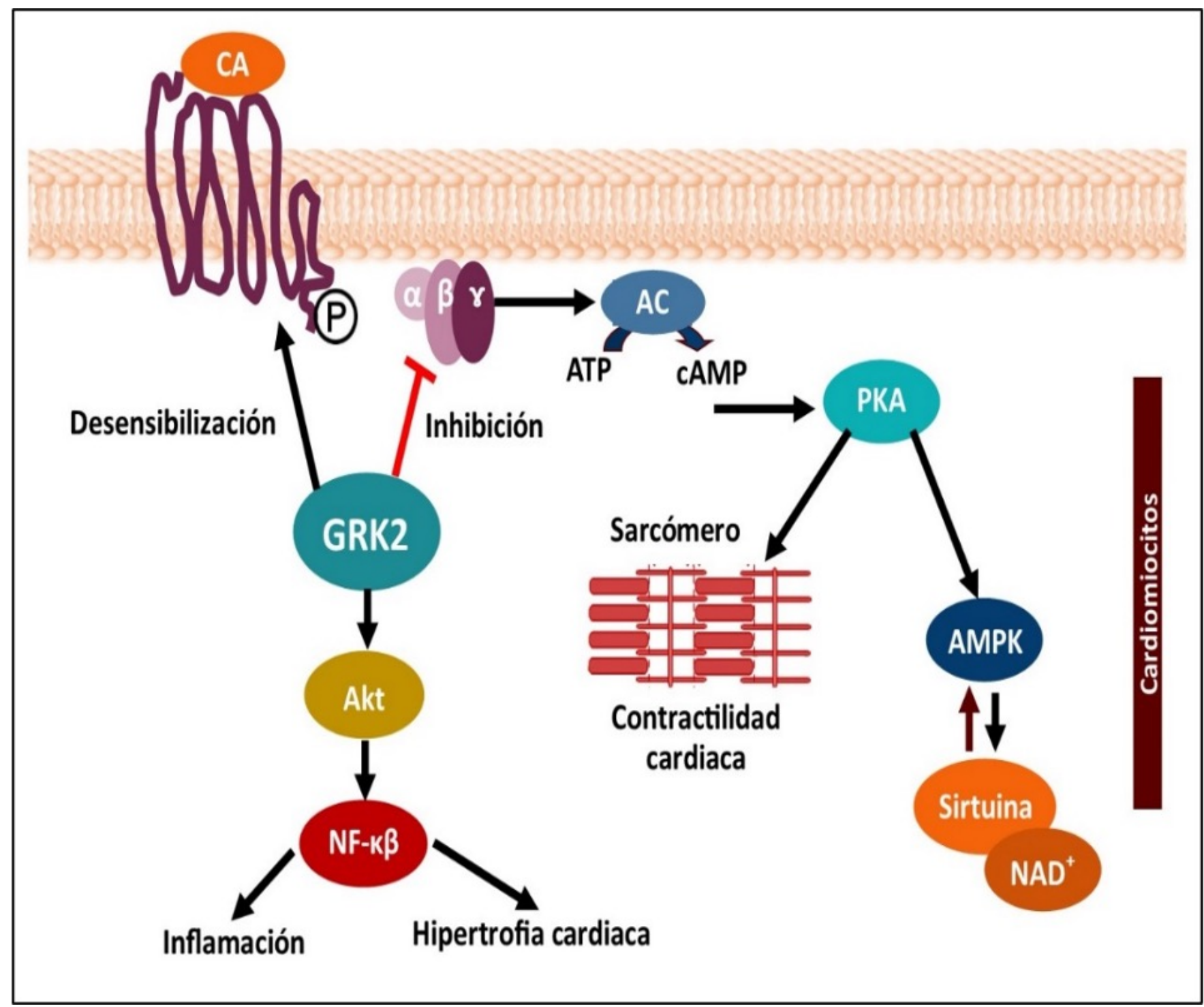

Figura 3. GRK2 en la falla cardiaca. Durante la falla cardiaca, el aumento de las catecolaminas circulantes se une a los $\beta A R$ en el miocardio y cooperan con la actividad mejorada de GRK2 para realizar una reducción significativa de los receptores y una función cardíaca disminuida. La expresión cardíaca específica de $\beta$ ARKct compite con GRK2 endógeno por la asociación con $\mathrm{G} \beta \gamma$, lo que da como resultado una menor translocación de membrana de GRK2 al receptor, y la normalización de la señalización de $\beta A R$ en los miocitos defectuosos, a través de esta capacidad de respuesta recuperada, se reduce la actividad del SNS y se puede mejorar la función cardíaca. $\beta$ AR: receptor $\beta$ adrenérgico, CA: catecolaminas, AC: adenilato ciclasa, PKA: proteína cinasa A, AMPK 5': proteína de adenosina activada por monofosfato cinasa. 


\section{$\mathrm{NF}-\kappa \beta$ en hipertrofia cardiaca}

Es un factor de transcripción de cadena $\kappa$ de anticuerpos de células $\beta$, llamado factor nuclear $\kappa \beta$ $(\mathrm{NF}-\kappa \beta)$, que parece jugar un papel clave en diversas patologías cardiacas como: hipertrofia, daño por isquemia repercusión y enfermedades que tienen en común la participación de tejido de musculo liso y endotelial (23).

La actividad del NF- $\kappa \mathrm{B}$ depende de su localización celular, si está en el citoplasma se encuentra formando un complejo trimétrico con alguno de sus inhibidores (IкB) que oculta sus secuencias de destino nuclear y es, por tanto, transcripcionalmente inactivo. Cuando el $\mathrm{I} \kappa \mathrm{B}$ se fosforila es ubiquitinilado, lo que le permite su reconocimiento por el proteosoma degradándolo, liberando al dímero del NF- $\kappa \beta$ que es un factor pleiotrópico. Se ha sugerido que podría constituir un segundo mensajero, hasta el momento se ha asociado a NF$\kappa \beta$ con la expresión de varias citocinas y moléculas de adhesión celular. Algunos trabajos han informado que la adiponectina inhibe la señalización hipertrófica en el miocardio, por tanto, la señalización del NF-k $\beta$ está asociada a la proteína activadora 1 (AP-1), y desencadena apoptosis. Estos resultados se han observado, usando como modelo cultivos primarios de cardiomiocitos murinos de neonatos in vitro, bajo condiciones de estrés oxidativo (23).

Este factor participa en la protección contra la apoptosis en células cardiacas, al haber falta de oxigenación del miocardio (hipoxia), uno de los efectores de esta respuesta anti-apoptótica es una citocina liberada de forma autocrina llamada cardiotropina 1; Sin embargo, para que esta molécula pueda actuar se requiere la translocación nuclear del NF- $\beta \beta$ (24).

Se ha logrado tener progresos significativos en esclarecer los mecanismos moleculares para demostrar el papel clave que tiene el NF- $\kappa \beta$ en algunas patologías cardíacas. Sin embargo, a diferencia de lo que ocurre con los cardiomiocitos en cultivo, donde NF- $\kappa \beta$ se destaca por su papel antiapoptótico, en algunas alteraciones cardíacas como la isquemia-reperfusión, el rechazo a trasplante, los síndromes coronarios agudos y la aterosclerosis, parece tener una función pro-apoptótica, sólo en el caso de la precondición isquémica tiene una función anti-apoptótica (25).

Un efecto importante en donde se ha detectado la participación de NF- $\kappa \beta$ es en la hipertrofia inducida por angiotensina II, la fenilefrina y la endotelina 1 provocando una estimulación de la actividad del NF$\kappa \beta$ que depende de la degradación de las proteínas I $\kappa \mathrm{B} \alpha$. Tal activación es necesaria para la producción de dos de los principales marcadores del fenómeno hipertrófico que son la producción del factor natriurético atrial (ANF) y el incremento de la talla de los cardiomiocitos, de hecho, la sobre expresión de las subunidades p65 o c-Rel provoca por sí misma un incremento del tamaño celular (25), (Figura 5).

Es importante destacar que la inducción de la hipertrofia también puede ocurrir por estimulación con el TNFa, en cardiomiocitos aislados y es mediada por la activación del NF- $\kappa \beta$ a través de la generación de ROS (26).

En el proceso de la hipertrofia ventricular izquierda (HVI) se ha visto que el NF- $\kappa \beta$ está involucrado en el incremento del tamaño celular no obstante en condiciones normales los cardiomiocitos pueden crecer de manera hipertrófica, un evento caracterizado por el incremento de tamaño celular es el contenido de proteínas contráctiles de genes embrionarios como el ANF. Purcell et al., fueron los primeros en demostrar que la activación del NF- $\kappa \beta$ es necesaria y suficiente para inducir una respuesta hipertrófica a través de proteínas $G$ acopladas a receptores en cardiomiocitos aislados, de igual manera Higuchi et al., demostraron que la inducción de hipertrofia por el TNF $\alpha$ en cardiomiocitos en cultivo es dependiente de la activación del NF- $\kappa \beta$ $(26,27)$.

A pesar de que la hipertrofia es una respuesta de naturaleza adaptativa y compensatoria cuando el corazón es sometido a exceso de trabajo puede llegar a ocasionar lesiones focales. Algo que ha impedido el avance del estudio in vivo de la hipertrofia es la 
carencia de modelos experimentales es por ello que aún muchos estudios no son concluyentes en cuanto a la relación que existe entre el NF- $\kappa \beta$, la hipertrofia cardiaca y por tanto los mecanismos responsables de la hipertrofia y la transición hacia las fallas cardiacas son pobremente atendidas (26).

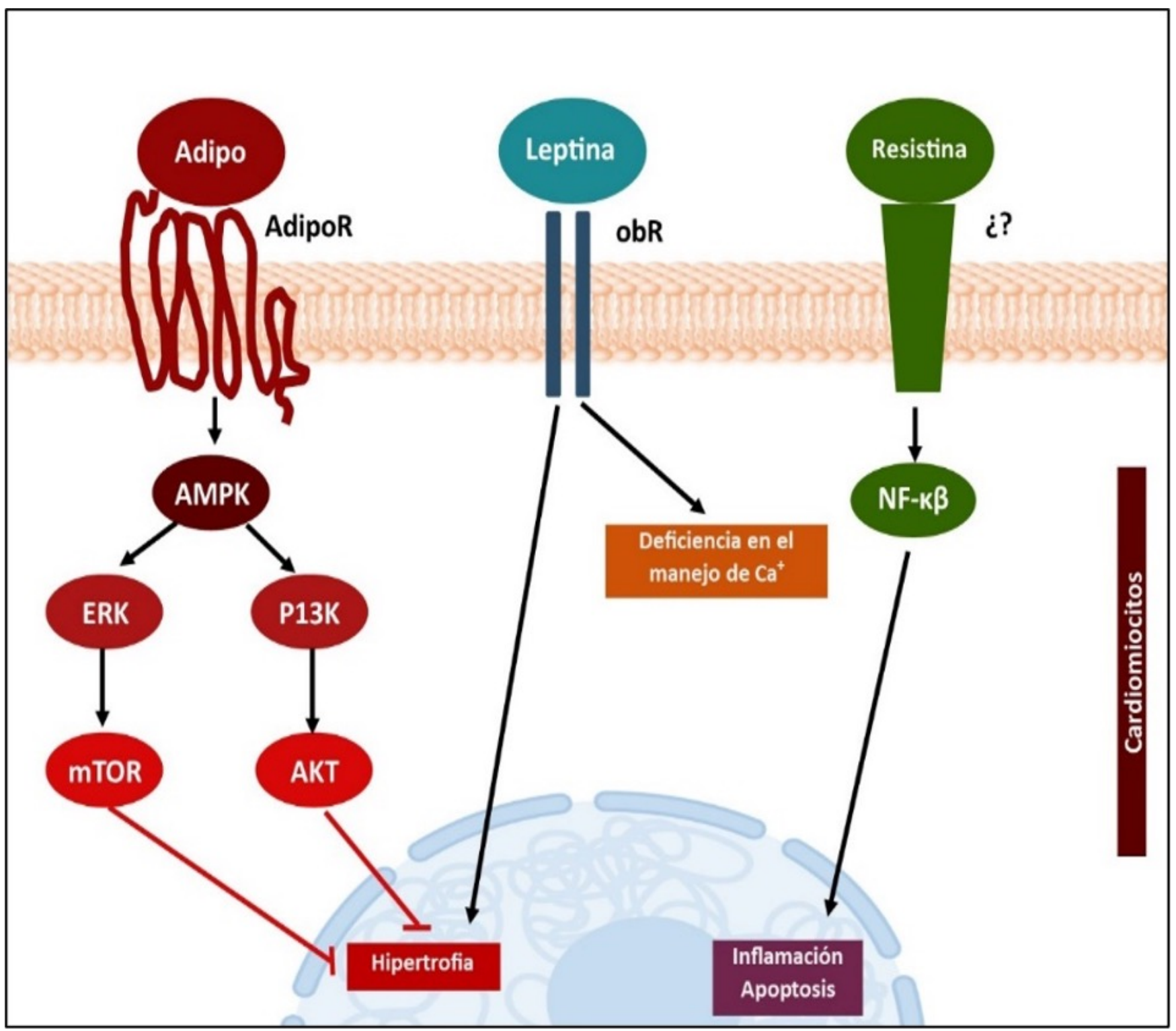

Figura 4. Vía de las adipocinas y su efecto en el cardiomiocito. Las adipocinas se difunden en la pared de los vasos coronarios actuando mediante un "mecanismo de señalización vasocrina". La adiponectina puede unirse a dos isoformas de receptores (AdipoR 1/2). Esta promueve la oxidación de ácidos grasos y la absorción de glucosa, además disminuye la síntesis de ROS inducida por glucosa. La regulación a la baja de la adiponectina y sus receptores en la obesidad está relacionada con la resistencia a la insulina y la deposición intramuscular de lípidos. Considerada una adipocina protectora, la adiponectina se asocia con cardioprotección además de acciones: antiinflamatorias, antioxidantes, antiaterogénicas y antiapoptóticas. La leptina, codificada por el gen de la obesidad (Ob), se une a receptores OrbR. Su secreción se correlaciona positivamente con el índice de masa corporal y tiene una producción más significativa en el TA subcutáneo que en el TA visceral. 


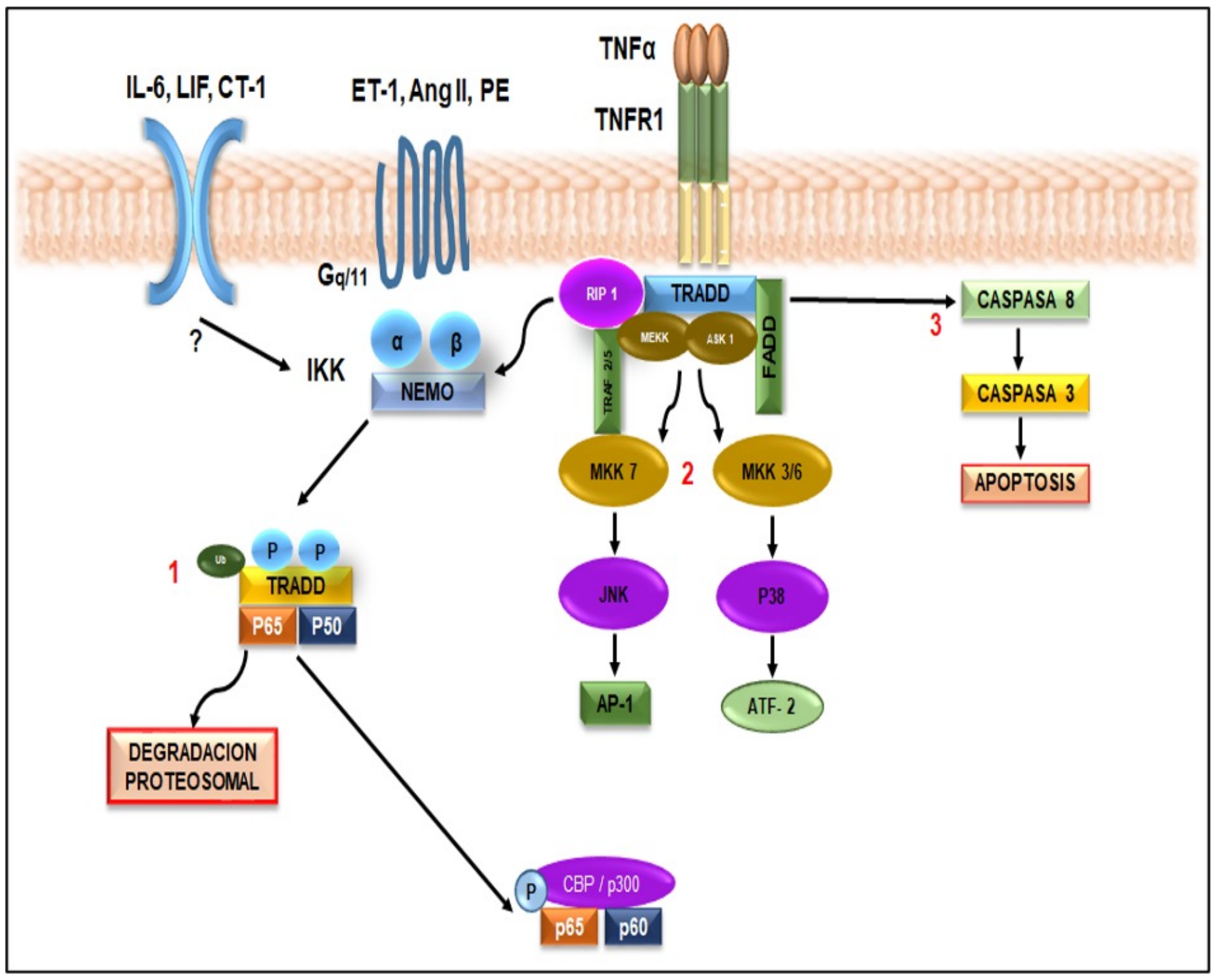

Figura 5. Las vías de señalización del TNFa y del NF-кB canónico. El ligando del receptor de TNF $\alpha$ (TNFR1) da como resultado el reclutamiento de la proteína de dominio de muerte asociada a TNFR (TRADD), que activa 3 vías de señalización definidas clásicamente: (1) señalización canónica de NF- $\mathrm{BB}$; (2) señalización JNK y p38 MAPK; y (3) activación de caspasa. En la señalización canónica de NF- $\kappa B$, el dímero de NF- $\kappa B$ compuesto por p65 y p50 se mantiene en un estado inactivo en el citosol por I $\kappa \mathrm{B} \alpha$. Tras la activación de TNFR1, los factores asociados al receptor de TNF (TRAF2 / 5) activan el complejo IKK, lo que da como resultado la fosforilación de IкB $\alpha$ y la posterior degradación

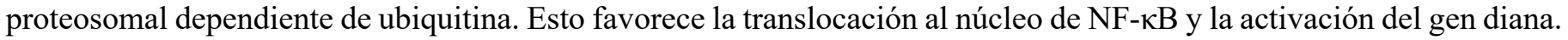
La posible sincronización y el contexto celular determinan el resultado de estas vías de señalización. Además, los agentes hipertróficos de la familia IL-6, así como los agonistas del receptor acoplado a Gq / 11 activan la señalización canónica de NF- $\kappa \mathrm{B}$; sin embargo, queda por evaluar el mecanismo por el cual estos agentes estimulan la degradación del $\mathrm{I} \kappa \mathrm{B} \alpha$ (factor inhibidor de la leucemia [LIF]; cardiotropina-1 [CT-1]; endotelina-1 [ET-1], fenilefrina [PE]). Modificado de Gordon, J. W., Shaw, J. A., \& Kirshenbaum, L. A. (2011). Multiple Facets of NF-кB in the Heart. Circulation Research, 108(9), 1122-1132. 


\section{Swiprosina -1, un sensor de Ca2+}

La Swiprosina-1 es una proteína que se codificada por el gen $\mathrm{EFhD} 2$ de unión al $\mathrm{Ca} 2+$ del citoesqueleto identificada en los mastocitos, el cerebro y el sistema inmune humano, el dominio EFhd2 se estructura con una región $\mathrm{N}$-terminal desordenada seguida de los factores de elongación (EF) y un dominio de bobina en espiral en el C-terminal; esta molécula modula la expresión de citocinas por remodelación de actina en mastocitos humanos, los sitios de EF están involucrados directamente en la agrupación de filamentos de actina (F-actina) dependiente de $\mathrm{Ca} 2+$ y el dominio de la bobina enrollada es esencial para la agrupación de F-actina por homodimerización (28).

Su papel principal es actuar como un sensor de $\mathrm{Ca} 2+$ ya que al encontrase niveles altos de este catión durante la diástole se activa la Swinprosina-1 promoviendo la formación de dímeros que estabilizan a F-actina bloqueando los sitios de unión de la cofilina en los cardiomiocitos(29), lo cual genera la presencia de estructuras similares a seudópodos en el postinfarto; estudios in situ, en cardiomiocitos ventriculares de rata adulta han confirmado la formación de estas estructuras similares a los seudópodos, evidenciando de esta manera la participación estimulante de la Swiprosina-1 necesaria para la formación de estructuras similares a seudópodos(30). Hasta ahora se ha propuesto que sin la acción de la swinprosina1 la RC no tendría lugar (31).

En la tabla 1 se citan algunas de las biomoléculas participantes en el mecanismo de RC.

\section{CONCLUSIÓN}

El desarrollo del SM y sus comorbilidades (principalmente la falla cardiaca) se encuentra acompañado de la modulación de diversas vías de señalización intracelular en los cardiomiocitos los cuales participan en la RC, generando cambios estructurales adaptativos a la dinámica fisiopatológica que son muy similares entre sí independientemente de si se presentan por una patología única o por el conjunto de estas que comprenden la totalidad del SM.

CONFLICTOS DE INTERESES: Los autores del presente trabajo no tenemos ningún conflicto de intereses con respecto a la información que se expone en el presente manuscrito.

\section{CONTRIBUCIÓN DE LOS PARTICIPANTES}

Vargas-López Misael.- Participo en: la búsqueda de información bibliográfica mediante servidores electrónicos institucionales, así como en la redacción del presente manuscrito, además del diseño y edición de las imágenes que se incluyen en el presente trabajo.

Edgar Fernando Cortes Martínez.- Participo en: la búsqueda de información para el apartado de nutrición, la presentación de la información de la tabla y la revisión de la redacción.

José Antonio Velázquez Domínguez.- Participo en la búsqueda de información bibliográfica, el análisis y estructuración de la edición del presente trabajo, así como en la dirección del mismo. 


\section{TABLA 1. CARACTERISTICAS DE LAS PRINCIPALES BIOMOLÉCULAS ASOCIADAS CON RC}

\begin{tabular}{|c|c|c|c|c|}
\hline NOMBRE & $\begin{array}{l}\text { INTERACCIÓN } \\
\text { CELULAR }\end{array}$ & FUNCIÓN & $\begin{array}{l}\text { REMODELACIÓN CARDIACA Y } \\
\text { EXPRESIÓN MOLECULAR }\end{array}$ & BIBLIOGRAFIA \\
\hline Gal - 3 & Sobreproducción de MEC & $\begin{array}{l}\text { Mediador potencial del daño } \\
\text { cardiaco. } \\
\text { Amplificación de moléculas } \\
\text { proinflamatorias }\end{array}$ & $\begin{array}{l}\text { Acción en miocardio y vasos sanguíneos, } \\
\text { presente en falla cardiaca. }\end{array}$ & $\begin{array}{l}\frac{1}{2} \text { Al-Daghri NM., et al., } \\
\frac{2}{2} \text { Tadic M., et al., } 2015 \\
\underline{3} \text { Xu Z., et al., } 2016\end{array}$ \\
\hline TGF - B1 & & Produce fibrosis miocárdica & Acción en miofibroblastos, presente en DM2. & $\begin{array}{l}\underline{4} \text { Martinez-Martinez E., et } \\
\text { al., } 2015 .\end{array}$ \\
\hline $\boldsymbol{\alpha - S M A}$ & Mediador de la MEC & Mediador del metabolismo & $\begin{array}{l}\text { Acción en miofibroblastos, también presente en } \\
\text { musculo liso. }\end{array}$ & $\begin{array}{l}\underline{1} \text { Al-Daghri NM. et al., } 2012 \\
\underline{2} \text { Tadic M., et al., } 2015 \\
\underline{3} \text { Xu Z., et al., } 2016\end{array}$ \\
\hline MMP & Degradación de la MEC & Fibrosis cardiaca & $\begin{array}{l}\text { A nivel cardiaco en pacientes diabéticos se } \\
\text { expresan las subfamilias MMP-2 y MMP-9 }\end{array}$ & $\begin{array}{l}\frac{1}{2} \text { Al-Daghri NM. et al., } 2012 \\
\frac{2}{3} \text { Tadic M., et al., } 2015 \\
\frac{3}{4} \text { Xu Z., et al., } 2016 \\
\underline{4} \text { Martinez-Martinez E., et } 2015 .\end{array}$ \\
\hline PIN 1 & $\begin{array}{l}\text { Regulación negativa de la } \\
\text { citosina fibrótica, la } \\
\text { proliferación, la migración } \\
\text { de FC y la modulación en } \\
\text { la expresión de MMP. }\end{array}$ & $\begin{array}{l}\text { Disminuye la inflamación de las } \\
\text { vías respiratorias, la deposición } \\
\text { de colágeno pulmonar en el } \\
\text { plasma y la dimetil-nitrosamina } \\
\text { inducida por la fibrosis hepática. }\end{array}$ & $\begin{array}{l}\text { En DM2, desencadena indirectamente la } \\
\text { señalización del TGF- } \beta\end{array}$ & $\begin{array}{l}\frac{1}{2} \text { Al-Daghri NM. et al., } 2012 \\
\frac{2}{3} \text { Tadic M., et al., } 2015 \\
\underline{\text { Xu Z., et al., } 2016}\end{array}$ \\
\hline P38 & $\begin{array}{l}\text { Miembro de la familia } \\
\text { MAPK. }\end{array}$ & $\begin{array}{l}\text { Regula la supervivencia y la } \\
\text { hipertrofia celular. }\end{array}$ & $\begin{array}{l}\text { Acción en cardiomiocitos, la subfamilia p } 38 \delta \text { lo } \\
\text { expresa tejido cardiaco sano; las subfamilias } \\
\text { p38 y p38 } \gamma \text { muestran menor expresión, p } 38 \alpha \\
\text { facilita la apoptosis. }\end{array}$ & \\
\hline $\begin{array}{l}\text { SWIPROSIN } \\
\text { / 1-EFhD-2 }\end{array}$ & Sensor de $\mathrm{Ca}^{2+}$ & $\begin{array}{l}\text { Participa en la formación de } \\
\text { estructuras similares a los } \\
\text { seudópodos }\end{array}$ & Aparece solo en condiciones de isquemia & 1 Al-Daghri NM. et al., 2012 \\
\hline GRK2 & $\begin{array}{l}\text { Modulador directo de la } \\
\text { insulina en varios tejidos. }\end{array}$ & $\begin{array}{l}\text { Induce la hipertrofia cardiaca en } \\
\text { la IC. }\end{array}$ & $\begin{array}{l}\text { Se encuentran niveles elevados en la primera } \\
\text { etapa de la cardiomiopatía. }\end{array}$ & $\begin{array}{l}\underline{1} \text { Al-Daghri NM. et al., } 2012 \\
\underline{2} \text { Tadic M., et al., } 2015\end{array}$ \\
\hline Adiponectina & $\begin{array}{l}\text { Potenciación de la acción } \\
\text { de la insulina, aumenta la } \\
\text { producción de óxido } \\
\text { nítrico, estimula la síntesis } \\
\text { de prostaglandinas y }\end{array}$ & $\begin{array}{l}\text { Modula las reacciones } \\
\text { antiinflamatorias e Inhibe la } \\
\text { remodelación del miocardio. }\end{array}$ & & $\underline{3} \mathrm{Xu}$ Z., et al., 2016 \\
\hline
\end{tabular}


participa en la activación de la adenosina monofosfato.

\section{Leptina \\ Efecto inotrópico negativo}

que induce hipertensión.

Resistina Se relaciona con la resistencia a la insulina y la dislipidemia.

JNK Miembro de la familia MAPK.

NF-KB

Se encuentra de forma inactiva en casi todas las células
Se encuentran niveles reducidos en personas con obesidad.

Estimula la hipertrofia cardíaca.

Inhibe la transportación vesicular de glucosa

Participan en la modulación de la acción de insulina y como componente patogénico en obesidad, hígado graso y DM2

Apoptótica y anti apoptótica dependiendo de su localización.
Se encuentra elevada en reacciones Inflamatorias, resistencia a la insulina y falla cardiaca.

Esta correlacionada por la actividad de la leptina, Las JNK 1- $\beta$ y 2 se expresan en varios tejidos mientras que la JNK3 únicamente se expresa en cerebro, islotes pancreáticos, corazón y testículo.

Se expresa cuando existe hipertrofia del cardiomiocito dañado por isquemia se expresa en musculo liso, tejido endotelial de vasos sanguíneos.
1 Al-Daghri NM. et al., 2012

2 Tadic M., et al., 2015

$3 \mathrm{Xu} \mathrm{Z}$., et al., 2016

4 Martinez-Martinez E., et al., 2015.

1 Al-Daghri NM. et al., 2012

2 Tadic M., et al., 2015

1 Al-Daghri NM. et al., 2012 


\section{REFERENCIAS}

1. Al-Daghri NM, Alkharfy KM, Al-Saleh Y, Al-Attas OS, Alokail MS, Al-Othman A, et al. Modest reversal of metabolic syndrome manifestations with vitamin $\mathrm{D}$ status correction: A 12-month prospective study. Metabolism [Internet].

2012;61(5):661-6. http://dx.doi.org/10.1016/j.metabol.2011.09.017

2. Tadic M, Cuspidi C. Childhood obesity and cardiac remodeling: From cardiac structure to myocardial mechanics. J Cardiovasc Med. 2015;16(8):538-46.

3. Xu Z, Sun J, Tong Q, Lin Q, Qian L, Park Y, et al. The role of ERK1/2 in the development of diabetic cardiomyopathy. Int J Mol Sci. 2016;17(12):1-17.

4. Martínez-Martínez E, López-Ándres N, Jurado-López R, Rousseau E, Bartolomé MV, Fernández-Celis A, et al. Galectin-3 participates in cardiovascular remodeling associated with obesity. Hypertension. 2015;66(5):9619.

5. De Boer RA, Van Der Velde AR. Galectin-3: A new biomarker for heart failure progression and prognosis. LaboratoriumsMedizin. 2013;37(5):251-60.

6. Bobronnikova L. Galectin-3 as a potential biomarker of metabolic disorders and cardiovascular remodeling in patients with hypertension and type 2 diabetes. Vessel Plus. 2017;1(2):61-7.

7. Yue $\mathrm{Y}$, Meng $\mathrm{K}, \mathrm{Pu} \mathrm{Y}$, Zhang $\mathrm{X}$. Transforming growth factor beta (TGF- $\beta$ ) mediates cardiac fibrosis and induces diabetic cardiomyopathy. Diabetes Res Clin Pract [Internet]. 2017;133:124-30. Available from: http://dx.doi.org/10.1016/j.diabres.2017.08.018

8. Liu G, Ma C, Yang H, Zhang PY. Transforming growth factor $\beta$ and its role in heart disease. Exp Ther Med. 2017; 13(5):2123-8.

9. Liu X, Liang E, Song X, Du Z, Zhang Y, Zhao Y. Inhibition of Pin 1 alleviates myocardial fibrosis and dysfunction in STZ-induced diabetic mice. Biochem Biophys Res Commun 2016;479(1):109-15. http://dx.doi.org/10.1016/j.bbrc.2016.09.050

10. Shaker YM, Soliman HA, Ezzat E, Hussein NS, Ashour E, Donia A, et al. Serum and urinary transforming growth factor beta 1 as biochemical markers in diabetic nephropathy patients. Beni-Suef Univ J Basic Appl Sci. 2014;3(1):16-23. http://dx.doi.org/10.1016/j.bjbas.2014.02.002

11. Huang GL, Qiu JH, Li B Bin, Wu JJ, Lu Y, Liu XY, et al. Prolyl isomerase pin1 regulated signaling pathway revealed by pin $1+/+$ and Pin 1 -/- mouse embryonic fibroblast cells. Pathol Oncol Res. 2013;19(4):667-75.

12. Turner, Blythe. Cardiac Fibroblast p38 MAPK: A Critical Regulator of Myocardial Remodeling. J Cardiovasc Dev Dis. 2019;6(3):27.

13. Muslin JA. MAPK Signaling in cardiovascular health and desease: Molecular mechanism and therapeutic targets. Clin Sci. 2009;115(7):203-18.

14. Wang S, Ding L, Ji H, Xu Z, Liu Q, Zheng Y. The role of p38 MAPK in the development of diabetic cardiomyopathy. Int J Mol Sci. 2016;17(7):1-14.

15. Craige SM, Chen K, Blanton RM, Keaney JF, Kant S. JNK and cardiometabolic dysfunction. Biosci Rep. 2019;39(7):1-18.

16. Pal M, Febbraio MA, Lancaster GI. The roles of c-Jun $\mathrm{NH} 2$-terminal kinases (JNKs) in obesity and insulin resistance. J Physiol. 2016; 594(2): 267-79.

17. Schumacher-Bass SM, Traynham CJ, Koch WJ. G protein-coupled receptor kinase 2 as a therapeutic target for heart failure. Drug Discov Today Ther Strateg. 2012;9(4):1-14.

18. Woodall MC, Ciccarelli M, Woodall BP, Koch WJ. GRK2 - A Link Between Myocardial Contractile Function and Cardiac Metabolism. Circ Res. 2014;114(10):1661-70.

19. Goncąlves N, Falcaõ-Pires I, Leite-Moreira AF. Adipokines and their receptors: Potential new targets in cardiovascular diseases. Future Med Chem. 2015;7(2):139-57.

20. Hui X, Lam KS, Vanhoutte PM, Xu A. Adiponectin and cardiovascular health: An update. Br J Pharmacol. 2012;165(3):574-90.

21. Imo A. E, David C. GJ, Carlos J. R, Haiying C, Alain G. B. Mechanisms of heart failure in obesity. Obes Res Clin Pr. 2014;8(6):e540-8.

22. Katsiki N, Mikhailidis DP, Banach M. Leptin, cardiovascular diseases and type 2 diabetes mellitus review-article. Acta Pharmacol Sin. 2018;39(7):1176-88. 
23. Fujioka S, Niu J, Schmidt C, Sclabas GM, Peng B, Uwagawa $\mathrm{T}$, et al. NF-k $\beta$ and AP-1 Connection: Mechanism of NF-k $\beta$ Dependent Regulation of AP-1 Activity. 2004;24(17):7806-19.

24. Craig R, Wagner M, McCardle T, Craig AG, Glembotski CC. The Cytoprotective Effects of the Glycoprotein 130 Receptor-coupled Cytokine, Cardiotrophin-1, Require Activation of NF-кB. J Biol Chem. 2001;276(40):376219.

25. Hernández-Gutiérrez S, Rojas-del Castillo E. edigraphic.com. El Pap del factor transcripción NF- $\mathrm{BB}$ en la célula cardíaca. 2005;75:363-70.

26. Higuchi M, Manna SK, Sasaki R, Aggarwal BB. Regulation of the activation of nuclear factor $\mathrm{\kappa B}$ by mitochondrial respiratory function: Evidence for the reactive oxygen species-dependent and -independent pathways. Antioxidants Redox Signal. 2002;4(6):94555.

27. Purcell NH, Tang G, Yu C, Mercurio F, DiDonato JA, Lin A. Activation of NF- $\kappa B$ is required for hypertrophic growth of primary rat neonatal ventricular cardiomyocytes. Proc Natl Acad Sci U S A. 2001;98(12):6668-73.

28. Park KR, Kwon MS, An JY, Lee JG, Youn HS, Lee Y, et al. Structural implications of $\mathrm{Ca} 2+$-dependent actinbundling function of human EFhd2/Swiprosin-1. Sci Rep. 2016;6(July):1-15. http://dx.doi.org/10.1038/srep39095

29. Huh YH, Kim SH, Chung KH, Oh S, Kwon MS, Choi HW, et al. Swiprosin-1 modulates actin dynamics by regulating the F-actin accessibility to cofilin. Cell Mol Life Sci. 2013;70(24):4841-54.

30. Schreckenberg R, Pöling J, Lörchner H. Swiprosin1/EFhD-2 Expression in Cardiac Remodeling and PostInfarct Repair: Effect of Ischemic Conditioning. 2020;2:1-13.

31. Nippert F, Schreckenberg R, Hess A, Weber M, Schlüter $\mathrm{KD}$. The effects of swiprosin-1 on the formation of pseudopodia-like structures and $\beta$ - adrenoceptor coupling in cultured adult rat ventricular cardiomyocytes. PLoS One. 2016;11(12):1-15. 